\title{
A Multicast Routing Protocol for Group Communications in Cellular IP Networks
}

\author{
Chun-Chuan Yang, Chun-Shian Tsai, and Kwin-Yee Lin \\ Multimedia and Communications Laboratory \\ Department of Computer Science and Information Engineering \\ National Chi Nan University, Taiwan, R.O.C. \\ ccyang@csie.ncnu.edu.tw
}

\begin{abstract}
In this paper, a novel location management scheme called Distributed Group Tracking (DGT) for group communications in Cellular IP networks is proposed. In DGT, Base stations track each member of a group and build a share multicast routing tree called DGT-Tree for the group in a distributed manner. Transmission of multicast packets among group members is along the group's DGT-Tree. Simulation study has demonstrated that a better performance can be achieved by DGT over the gateway-based counterpart in terms of transmission cost as well as link load balance.
\end{abstract}

Keywords: Multicast, Cellular IP, Mobility Management

\section{INTRODUCTION}

Mobility management at IP layer [1-3] is an essential component in wireless mobile networking. Mobile IP [4, 5] was proposed to support global Internet mobility through the introduction of location directories and address translation agents. On the other hand, Cellular IP [6-8] provides local mobility and handoff support for frequently moving hosts. Integration of Mobile IP and Cellular IP has been addressed in [9-11]. The basic idea of the integration is using the two protocols at the same time but in different levels. That is, Mobile IP is used for inter-subnet mobility (macro-mobility), while Cellular IP is employed for the intra subnet mobility (micro-mobility).

It was pointed out in our previous work [12] that the handoff and routing mechanisms in Cellular IP require all the data packets to be routed to the gateway before being routed to the destination. It results in a bad consequence that for internal traffic of which the packets are transmitted by a mobile host in the Cellular IP network and destined to another mobile host in the same Cellular IP network, the traffic is still routed to the gateway first, even the destination of the traffic is connecting to the same base station as the sender or a neighboring base station. It implies that the gateway is inevitably becoming a hot spot of traffic and therefore results in the phenomenon of load unbalance among wired links in the Cellular IP Network. To remedy the problem incurred by the original Cellular IP handoff and routing scheme, Distributed Mobile Tracking (DMT) was proposed [12].

This work was supported in part by the National Science Council, Taiwan, R.O.C., under grant NSC 93-2219-E-260-004
In DMT, the trace of an active mobile host is recorded and transformed to a routing tree (namely MT-Tree) by base stations. Base stations maintain the MT-Tree of a mobile host in a distributed manner, and each branch in the MT-Tree leads to the current position of the mobile host. Therefore, transmission of IP packets destined to a mobile host can follow the route (tree branch) provided by the host's MT-Tree instead of using the original gateway-based routing scheme. Simulation results had demonstrated a better performance of DMT than the original routing scheme in Cellular IP in terms of link load balancing and routing efficiency.

A multicast extension of DMT called Mobile Tracking-based Multicast Protocol (MTMP) was also proposed in our previous work. MTMP uses MT-Tree routing as long as the source base station finds that MT-Tree routing is available for all receivers of the destined group. Otherwise, the multicast traffic is forwarded to the gateway, which is also the manager of group membership information. The gateway is responsible for transmitting the packets to all receivers. The gateway-based multicasting was named GBMP-RO (Gateway-based Multicast Protocol with Route Option) in which an IP option was defined to carry addresses of the group members to which the multicast packet should be forwarded. Simulation studies had shown the effect of load balancing as well as the low transmission cost by MTMP. However, as the group size increases, the hit ratio of MT-Tree routing in MTMP decreases drastically that makes MTMP behave more like GBMP-RO.

The major reason that DMT does not performs so well in MTMP as in the unicast case is because DMT tracks each individual mobile host independently. Since the MT-Trees of all members in a same group are independently maintained, the chances of taking advantage of MT-Tree routing are slim for a large group. In order to cope with the problem of DMT in multicast transmission, a new scheme of mobile tracking, which is called Distributed Group Tracking (DGT), is proposed in this paper. Unlike DMT, DGT builds and maintains a shared routing tree called DGT-Tree for all members in a group. The DGT-Tree of a group connects all members in the group such that a multicast packet originated from one of the members can be routed to all the other members along the group's DGT-Tree. Therefore, DGT is specially designed for group 
DGT-Cache Structure:

\begin{tabular}{|l|l|l|}
\hline Group ID & Branch information & Radio user count $(R C)$ \\
\hline
\end{tabular}

(a) Structure of DGT-Cache

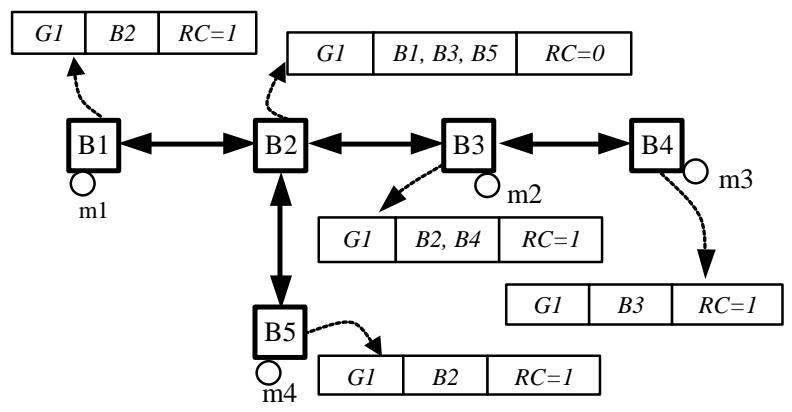

(b) Example of DGT-Tree (G1: m1, m2, m3, m4)

Figure 1. Using DGT-Cache for representing DGT-Tree

communications in which only the members in a group are senders and receivers of the group.

The rest of the paper is structured as follows. The basic idea of Distributed Group Tracking is explained in section II. Mechanisms for maintaining DGT-Tree are presented in section III. Simulation environment and results for performance evaluation are presented in section IV. Finally, section $\mathrm{V}$ concludes this paper.

\section{Distributed Group Tracking (DGT)}

\section{A. Basic idea}

The main idea of DGT is to build a shared multicast routing tree (DGT-Tree) among group members such that multicast packets transmitted by any of the members are routed to other members along the tree. Each link (branch) on a DGT-Tree is thus bi-directional. A new cache called DGT-Cache is defined in DGT. DGT-Cache is used to represent the branches of a DGT-Tree. Base stations in the Cellular IP network track all members in a group concurrently for maintaining DGT-Cache for the group's DGT-Tree in a distributed manner.

The data structure of DGT-Cache is shown in Figure 1-(a), in which three fields are defined: Group ID, Branch information, and Radio User Count (denoted by RC). Group ID records the identification of the group. Branch information records the branches of the DGT-Tree that are connecting to the base station. Branch information is a list of base station ID. RC records the number of group members that are currently connecting to the base station via radio interface. For instance, DGT-Cache entry $\left(G 1: B_{y}\right.$, $\left.B_{z}: R C=1\right)$ in base station $B_{x}$ means that there are two branches on Gl's DGT-Tree connecting to base station $B_{x}$ : branch $B_{x}-B_{y}$ and branch $B_{x}-B_{z} . R C=1$ means there is currently one group member connecting to base station $B_{x}$ via wireless interface. Note that we require the branches on a DGT-Tree to be bi-directional. Thus, there must be a DGT-Cache entry for $G 1$ in base stations $B_{y}$ and $B_{z}$ containing information for branches $B_{y}-B_{x}$ and $B_{z}-B_{x}$. An example for using DGT-Cache to represent a DGT-Tree is illustrated in Figure 1-(b).

DGT-Tree provides an easy way for multicast transmission among group members. For example, if mobile user $m 4$ in Figure 1-(b) wants to transmit a multicast packet to other members in $G 1$, it does so via its radio link to base station $B 5$. On receiving the multicast packet destined to group $G 1$, base station $B 5$ realizes from the DGT-Cache entry for $G 1$ that there is a branch connecting to $B 2$ and thus $B 5$ forwards the packet to $B 2$. Similarly, $B 2$ forwards the packet to $B 1$ and $B 3$. $B 3$ forwards the packet to $B 4$. Since $B 1, B 3$ and $B 4$ contain G1's DGT-Cache entry with non-zero RC value, they all broadcast the packet to each group member via radio interface.

\section{B. Constructing DGT-Tree}

In DGT, the gateway in a Cellular IP network is responsible for group membership management. To create a new group, a mobile user has to send a group initiation request with the ID of the group to the gateway. After the gateway successfully creates the new group, identification of the initiator of the group is recorded in the group membership table. The DGT-Tree for the new group at this time contains a single base station that the initiator is connecting to, and the DGT-Cache for the group created by the base station is (Group ID : Null : $R C=1$ ).

When a mobile user wants to join a group, it sends an IGMP-Join message to the gateway. If the join request is granted, the DGT-Tree for the group must be extended to include the current location of the new member. The current base station of the new member must grow branches until the new branches connect to the group's DGT-Tree. The problem is how does the new member know the correct way in which new branches should grow? To provide the correct way for tree growing, the gateway asks one of the group members (e.g. the initiator) to report its current base station (as the target base station for tree growing) to the new member. The new member then invokes DGT-Tree-Grow process toward the target base station.

Another problem arises: what if the member providing the target base station moves to another base station during DGT-Tree-Grow process such that the target base station is no longer on the group's DGT-Tree? The problem can be solved easily as explained in the following. When a base station is being removed from a group's DGT-Tree, we do not immediately clear the corresponding DGT-Cache entry; instead we merely mark the cache entry as invalid and set a garbage collection timer that is longer than the maximum 


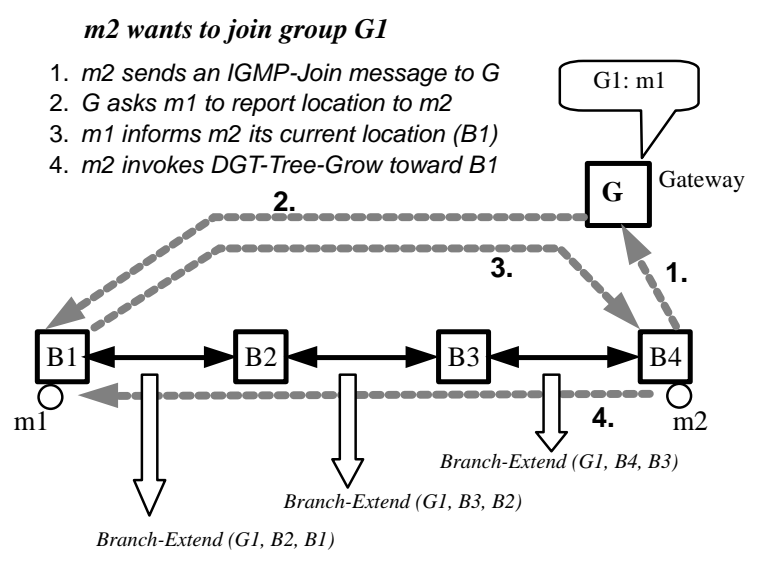

Figure 2. E.g. DGT-Tree-Grow process

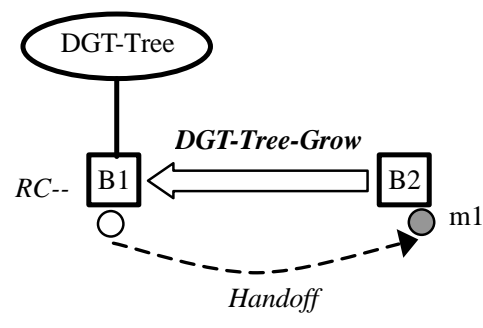

(a) New base station is not on DGT-Tree

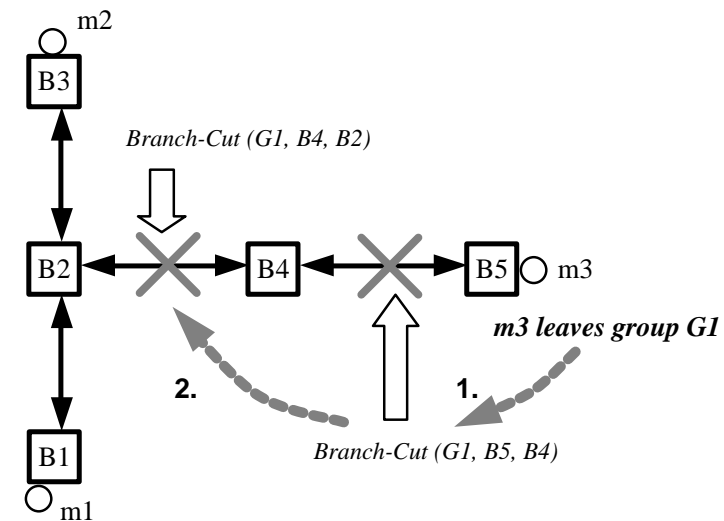

Figure 3. E.g. DGT-Tree-Trim process

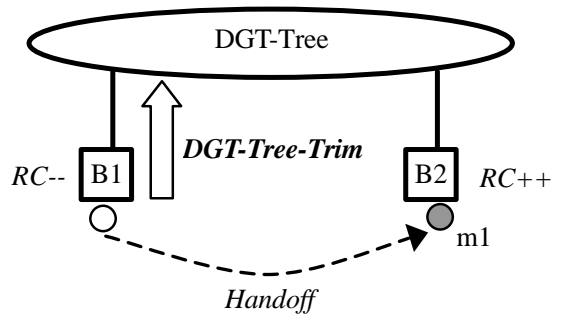

(b) New base station is on DGT-Tree

Figure 4. DGT handoff scheme

time to finish DGT-Tree-Grow process. Thus, even if the target base station is not on the group's DGT-Tree, we can follow the invalid cache entry to re-connect the target base station to the group's DGT-Tree. One example of DGT-Tree-Grow process is given in Figure 2.

In Figure 2, the first step for mobile user $m 2$ to join group $G 1$ is to send an IGMP-Join message to the gateway. After granting the request, the gateway asks the only member $m l$ of group $G l$ to report its current base station (Bl) to $m 2$. When receiving the IP address of $m l$ 's current base station, $m 2$ invokes DGT-Tree-Grow process started from $m 2$ 's current base station $B 4$ to the target base station $B 1$. As shown in Figure 2, DGT-Tree-Grow process is a series of branch creation operations. The operation of creating an individual branch between two base stations is called Branch-Extend operation in the paper. Branch-Extend operation involves updating corresponding DGT-Caches in the base stations on both ends of the branch. DGT-Tree-Grow process stops when the newly created branch has connected to the DGT-Tree, i.e., the newly created branch has connected to a base station that is already on the DGT-Tree.

On the other hand, another process called DGT-Tree-Trim process is invoked when a member leaves its group. As illustrated in Figure 3, DGT-Tree-Trim process removes branches (B5-B4 and $B 4-B 2)$ on the DGT-Tree that are no longer needed after group member $m 3$ has leaved the group. The operation of removing a single branch from a DGT-Tree is called Branch-Cut operation in the paper.

\section{MAINTAINING DGT-TREE}

\section{A. Dealing with mobility}

The DGT-Tree of a group has to adapt to the mobility of its mobile members, i.e. after the successful handoff of a mobile member to another base station, the group's DGT-Tree should be updated to match the new situation. In order to provide necessary information for updating DGT-Tree, a mobile member after handoff informs the new base station of the old base station's ID and informs the old base station of the new base station's ID.

As illustrated in Figures 4-(a) and 4-(b), there are two cases for updating the DGT-Tree after the handoff of a group member. In Figure 4-(a), mobile member $m l$ of a group moves from base station $B 1$ to another base station $B 2$ and $B 2$ is not on the group's DGT-Tree (i.e. no DGT-Cache entry existed for the group in $B 2$ ). $B 2$ invokes DGT-Tree-Grow process to perform a series of Branch-Extend operations to target base station $B 1$. On the other hand shown in Figure 4-(b), if $B 2$ is already on the group's DGT-Tree, there is no need for DGT-Tree-Grow process. But $B I$ has to check if the handoff resulting in unnecessary branches in the DGT-Tree. If so, $B 1$ invokes 


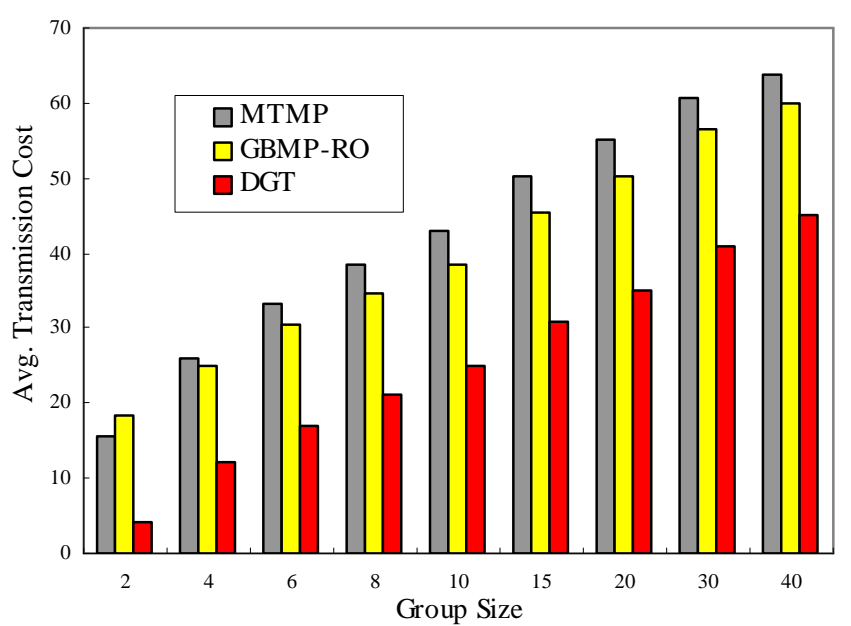

Figure 5. Average transmission cost $($ MoveProb $=0.5)$

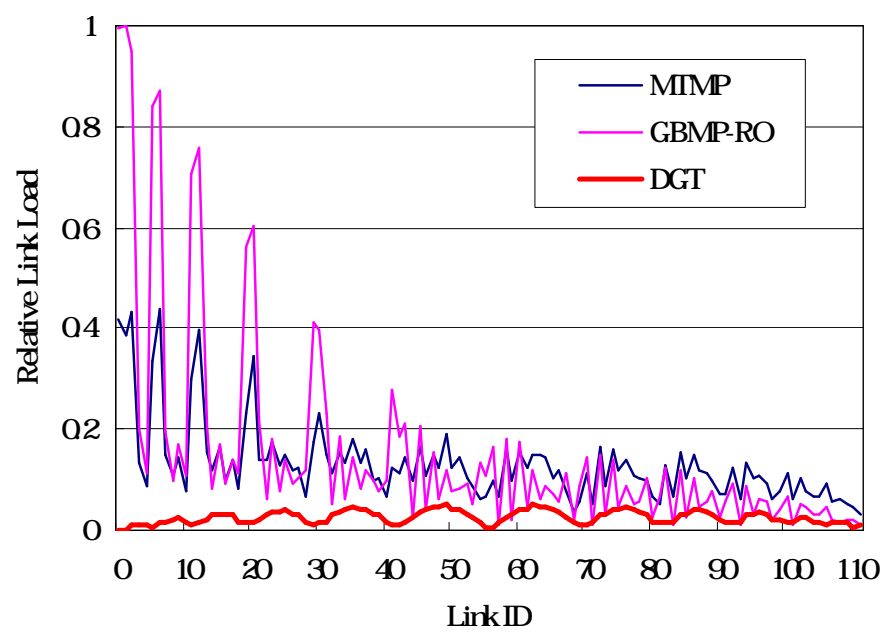

Figure 6. Relative link load at $600^{\text {th }}$ time slot (Group size $\left.=2\right)$

DGT-Tree-Trim process to cut unnecessary branches.

\section{B. Consistency of DGT-Cache}

As presented in previous paragraphs, two mechanisms to maintain DGT-Tree for handoff, joining, or leaving of a group member are DGT-Tree-Grow and DGT-Tree-Trim processes. Each of them uses Branch-Extend operation or Branch-Cut operation for updating DGT-Tree. Note that a branch in the DGT-Tree is bi-directional and both sides (base stations) of the branch maintain a DGT-Cache entry for the group. Therefore, in order to maintain the consistency of DGT-Cache data in Branch-Extend or Branch-Cut operations, updating the DGT-Cache entries for a branch in both base stations must be atomic. That is, there must not be two or more processes concurrently updating the DGT-Cache entries for a same branch. One possible solution to provide atomicity of updating DGT-Cache in related base stations of a branch is to lock corresponding DGT-Cache entries before updating them. Moreover, in

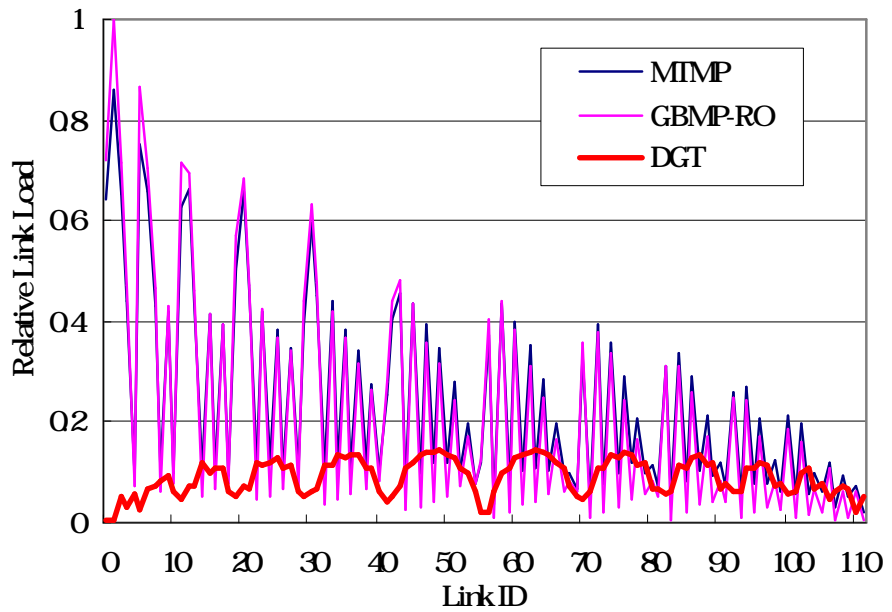

Figure 7. Relative link load at $600^{\text {th }}$ time slot $($ Group size $=10)$

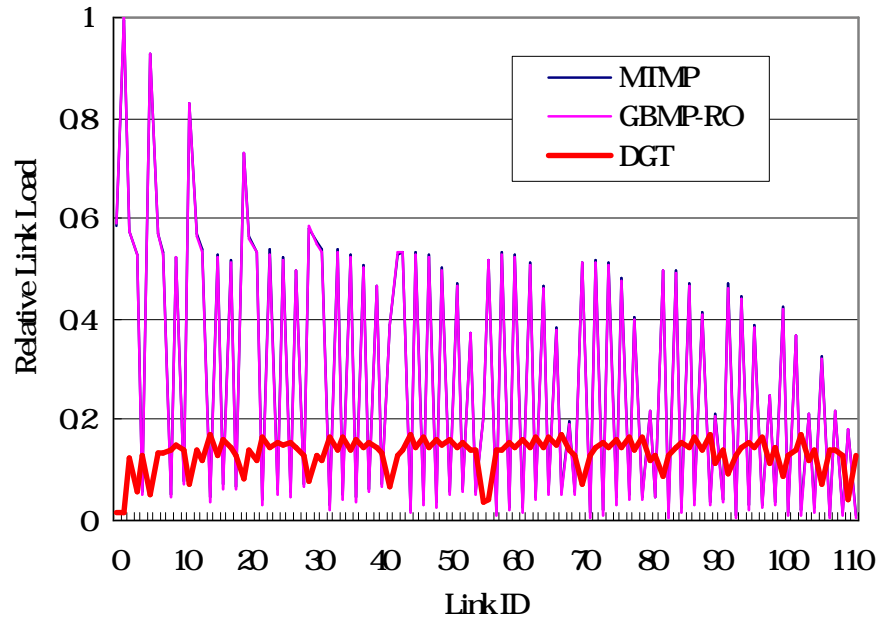

Figure 8. Relative link load at $600^{\text {th }}$ time slot $($ Group size $=40)$

order to get rid of deadlock situations, a process must concurrently lock the DGT-Cache entries of a branch in both base stations. If concurrent locking fails, the process has to wait a random time before performing another locking.

\section{Performance Evaluation}

\section{A. Simulation environment and performance criteria}

The network topology for the Cellular IP Network in the simulation is an $8 \times 8$ mesh. Each node in the mesh represents a base station and the gateway is located at the corner of the mesh. There are 5,000 mobile hosts in the network. Initial locations for the hosts are randomly selected from the base stations. In order to model the mobility of the hosts, time is slotted and a parameter called MoveProb (Movement Probability) is used in the simulation. MoveProb represents the probability that a mobile host leaves its current base station in the next time slot. Thus, 
we can model high mobility of hosts by assigning a large value of MoveProb. When a mobile host decides to leave the current base station in the next time slot, its next base station is randomly selected from the neighboring base stations.

To evaluate the performance of DGT, we create 1,000 groups and build corresponding DGT-Tree with a given group size. Group members are randomly selected from mobile hosts in the network in the beginning of the simulation. In each time slot, we randomly select a member from each group and the selected member transmits a multicast packet to its own group. Total run time in the simulation is 600 time slots. Two performance criteria are defined for comparing the performance of DGT with other schemes:

(1) Average transmission cost

(2) Relative load of each wired link

The transmission cost is defined as the total number of data packets generated in the Cellular IP network to transmit a packet to all group members. Relative load of each wired link is calculated as follows. The number of data packets transmitted in each wired link is recorded during the simulation. At the end of the simulation, the load of a wired link is computed as the number of packets transmitted in the link divided by the maximum number of packets among all wired links. That is, the load of the link with the maximum number of packets transmitted is set to 1 , and the load of other links is calculated by normalizing the number of packets to the maximum number of packets.

\section{B. Simulation results}

Average costs of group communications for MTMP, GBMP-RO, and DGT are displayed in Figure 5. It shows that proposed DGT outperforms the other two protocols in terms of average transmission cost. Moreover, Figure 5 also shows that DGT can save up to $50 \%$ of the transmission cost over GBMP-RO for group size under 4. For a large group size (e.g. 40), $25 \%$ of the transmission cost can be saved by DGT.

Figures 6 8 display the relative load of each wired link in the network for different group communications schemes with group sizes 2,10 , and 40 respectively. These figures demonstrate the better effect of load balancing as well as the efficiency of group communications by DGT.

Please note that since the simulation results for different MoveProb values are quite close, we only display the case of MoveProb $=0.5$ in the paper.

\section{Conclusion}

In this paper, a novel location management scheme and multicast routing protocol for group communications in Cellular IP networks are proposed. The location management scheme for a group of members is called
Distributed Group Tracking (DGT), which is based on location tracking of each member in a group. In DGT, Base stations track each member of a group and build a share routing tree called DGT-Tree for the group in a distributed manner. Transmission of multicast packets among group members is along the group's DGT-Tree. Simulation study has demonstrated that a better performance can be achieved by DGT over the gateway-based counterpart in terms of transmission cost as well as link load balance. Future work of the research includes more investigation on properties of DGT-Tree and overhead analysis of DGT.

\section{REFERENCES}

[1] K.W. Ng and V.C.M. Leung, "An IPv6-based Location Management Scheme for Client-Server Computing over Mobile Data Networks," Proc. IEEE Wireless Communications and Networking Conference (WCNC), 1999, pp. 525-529.

[2] T. Tung and A. Jamalipour, "Location Management Strategies for Wireless Networks- A Comparison Study on System Utilization," Proc. IEEE International Conference on Communications (ICC), 2001

[3] F.M. Chiussi, D.A. Khotimsky, and S. Krishnan, "Mobility management in third-generation all-IP networks," IEEE Communications Magazine, vol. 40, 2002, pp. 124-135.

[4] Charles Perkins, editor, "IP Mobility Support," Internet RFC 2002, Oct. 1996.

[5] T. F. La Prota, L. Salgarelli, G. T. Foster, "Mobile IP and Wide Area Wireless Data," Proc. IEEE Wireless Communications and Networking Conference (WCNC) 1999, pp. $1528-1532$.

[6] A.T. Campbell and J. Gomez, "An Overview of Cellular IP," Proc. IEEE Wireless Communications and Networking Conference (WCNC), 1999, pp. 606-610.

[7] A.T. Campbell, J. Gomez, S. Kim, A.G. Valko, C.Y. Wan, and Z.R. Turanyi, "Design, Implementation, and Evaluation of Cellular IP,” IEEE Personal Communications, Aug. 2000, pp. 42-49.

[8] G. Edwards and N. Suryakumar, "Cellular IP performance," Proc. IEEE Wireless Communications and Networking Conference (WCNC) 2003, pp. 2081-2085

[9] M. Carli, A. Neri, and A. R. Picci, "Mobile IP and Cellular IP Integration for Inter Access Networks Handoff," Proc. IEEE International Conference on Communications (ICC), 2001.

[10] K.D. Wong, "Architecture alternatives for integrating Cellular IP and Mobile IP," Proc. 21st IEEE International Performance, Computing, and Communications Conference 2002, pp. 197-204.

[11] J.-S. Chiang, Y.-H. Wang, C.-H. Tsai, and C.-P. Hsu, "Location management and multimedia communication service based on mobile IP and cellular IP network", Proc. 17th International Conference on Advanced Information Networking and Applications (AINA) 2003, pp. 223-226.

[12] Chun-Chuan Yang and Kwin-Yee Lin, "Distributed Mobile Tracking: A Novel Location Management Scheme for Routing Improvement in Cellular IP Networks," Journal of Computer Networks, vol. 43, issue 2, October 2003, pp. 147-161. 